\title{
Linear Momentum Conservation in the Motion of Electric Charges
}

\author{
Farrin Payandeh \\ Department of Physics, Payame Noor University (PNU), Tehran, Iran
}

Email address:

payandehfarrin92@gmail.com

\section{To cite this article:}

Farrin Payandeh. Linear Momentum Conservation in the Motion of Electric Charges. American Journal of Physics and Applications. Vol. 3, No. 2, 2015, pp. 60-62. doi: 10.11648/j.ajpa.20150302.17

\begin{abstract}
In this letter I will discuss the linear momentum conservation for an electric charge which is moving in a magnetic field. This will enrich the knowledge of undergraduate physics students, about the important concept of conservation of linear momentum, in classical electrodynamics.
\end{abstract}

Keywords: Conservation Laws, Lagrangian Mechanics, Electric Charges

\section{Introduction}

In Newtonian mechanics, the law of conservation of momentum can be derived from the law of action and reaction, which states that every force has a reciprocating equal and opposite force. Under some circumstances one moving charged particle can exert a force on another without any return force [1-6]. Moreover, Maxwell's equations, the foundation of classical electrodynamics, are Lorentzinvariant. Nevertheless, the combined momentum of the particles and the electromagnetic field is conserved.

Two electric charges $q_{1}$ and $q_{2}$ corresponding to masses $m_{1}$ and $m_{2}$ are supposed to be moving with velocities $\vec{v}_{1}$ and $\vec{v}_{2}$ in 3-dimensional space. As it is known from electromagnetism, reciprocal electric and magnetic forces are exerted on these two charges. According to this situation, the linear momentum conservation for particles in Coulomb potentials has been investigated and solved [7-11]. However in magnetic fields, this concept still appears to be obscure, since despite of the fact that the exerted magnetic forces on the charge are equal, they are not aligned in a same direction.

To obtain the conservation of linear momentum in this situation, it is sufficient to apply the Lagrangian mechanics. Assume $G$ to be a function of $t_{i}, p_{i}$ and $q_{i}$ such that

$$
G=G\left(p_{i}, t_{i}, q_{i}\right) .
$$

So the time derivative of this function becomes

$$
\frac{\mathrm{d} G}{\mathrm{~d} t}=\frac{\partial G}{\partial t}+\sum_{i}\left(\frac{\partial G}{\partial p_{i}} \frac{\partial p_{i}}{\partial t}+\frac{\partial G}{\partial q_{i}} \frac{\partial q_{i}}{\partial t}\right)
$$

The Hamilton equations imply that

$$
\begin{aligned}
& \frac{\partial H}{\partial q_{i}}=-\frac{\mathrm{d} p_{i}}{\mathrm{~d} t}, \\
& \frac{\partial H}{\partial p_{i}}=\frac{\mathrm{d} q_{i}}{\mathrm{~d} t} .
\end{aligned}
$$

According to (2) we have

$$
\frac{\mathrm{d} G}{\mathrm{~d} t}=\frac{\partial G}{\partial t}+\sum_{i}\left(\frac{\partial G}{\partial q_{i}} \frac{\partial H}{\partial p_{i}}-\frac{\partial G}{\partial p_{i}} \frac{\partial H}{\partial q_{i}}\right)
$$

Let us notate

$$
\{G, H\} \equiv \frac{\partial G}{\partial q_{i}} \frac{\partial H}{\partial p_{i}}-\frac{\partial G}{\partial p_{i}} \frac{\partial H}{\partial q_{i}}
$$

which is the Poisson's bracket. If $G$ is not an explicit function of time, then

$$
\frac{\mathrm{d} G}{\mathrm{~d} t}=\{G, H\}
$$

In the case of a vanishing Poisson's bracket, $G$ is a constant of motion in this physical system. Now to see what really happens in this physical process, in the next section, we deal with the conservation of the linear momentum for 
these charges by writing the usual Hamiltonian of two moving electric charges in a magnetic field.

\section{Obtaining the Conservation of Momentum}

Let us write the Hamiltonian for a two-particle system, consisting of the electric charges $q_{1}$ and $q_{2}$. As we know, the Hamiltonian of a charged particle in a magnetic field is $H=\frac{1}{2 m}\left(\vec{p}+\frac{e}{c} \vec{A}\right)^{2}$, which for a two-particle system becomes

$$
H=\frac{1}{2 m_{1}}\left(\vec{p}_{1}+\frac{q_{1}}{c} \vec{A}_{21}\right)^{2}+\frac{1}{2 m_{2}}\left(\vec{p}_{2}+\frac{q_{2}}{c} \vec{A}_{12}\right)^{2} .
$$

To obtain the vector potentials $\vec{A}_{12}$ and $\vec{A}_{21}$, let us note that $\vec{B}_{12}$, i.e. the magnetic field felt by $q_{1}$ which is produced by $q_{2}$ is (see figure 1 )

$$
\vec{B}_{12}=\frac{\vec{v}_{2}}{c} \times \frac{\vec{E}_{2}}{c}=\frac{q_{2}}{4 \pi \varepsilon_{0} c^{2}} \vec{v}_{2} \times \frac{\vec{r}_{1}-\vec{r}_{2}}{\left|\vec{r}_{1}-\vec{r}_{2}\right|^{3}}=\frac{q_{2}}{4 \pi \varepsilon_{0} c^{2}} \vec{v}_{2} \times \vec{\nabla}_{1} \frac{1}{\left|\vec{r}_{2}-\vec{r}_{1}\right|},
$$

or

$$
\begin{gathered}
\vec{B}_{12}=\vec{\nabla}_{1} \times\left(\frac{q_{2}}{4 \pi \varepsilon_{0} c^{2}} \frac{\vec{v}_{2}}{\left|\vec{r}_{2}-\vec{r}_{1}\right|}\right)=\vec{\nabla}_{1} \times \vec{A}_{12} . \\
H=\frac{1}{2 m_{1}}\left(\vec{p}_{1}+\frac{q_{1} q_{2}}{4 \pi \varepsilon_{0} c^{3}} \frac{\vec{v}_{2}}{\left|\vec{r}_{2}-\vec{r}_{1}\right|}\right)^{2}+\frac{1}{2 m_{2}}\left(\vec{p}_{2}+\frac{q_{1} q_{2}}{4 \pi \varepsilon_{0} c^{3}} \frac{\vec{v}_{1}}{\left|\vec{r}_{2}-\vec{r}_{1}\right|}\right)^{2} .
\end{gathered}
$$

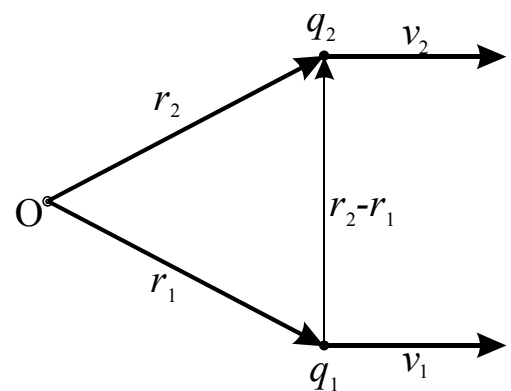

Figure 1. The vector difference between to moving charges $q_{1}$ and $q_{2}$, respectively of velocities $v_{1}$ and $v_{2}$.

Therefore according to the relation between the magnetic field and vector potential, $\vec{B}=\vec{\nabla} \times \vec{A}$, one obtains

$$
\vec{A}_{12}=\frac{q_{2}}{4 \pi \varepsilon_{0} c^{2}} \frac{\vec{v}_{2}}{\left|\vec{r}_{2}-\vec{r}_{1}\right|}
$$

and similarly

$$
\vec{A}_{21}=\frac{q_{1}}{4 \pi \varepsilon_{0} c^{2}} \frac{\vec{v}_{1}}{\left|\vec{r}_{2}-\vec{r}_{1}\right|}
$$

Substituting (9) and (10) in (6) we have

Now we interpolate this relation in the Poisson's bracket $\{\vec{p}, H\}$, where $\vec{p}=\vec{p}_{1}+\vec{p}_{2}$. We get

$$
\{\vec{p}, H\}=\left\{\vec{p}_{1}+\vec{p}_{2}, H\right\}=\frac{\partial\left(\vec{p}_{1}+\vec{p}_{2}\right)}{\partial q_{i}} \frac{\partial H}{\partial p_{i}}-\frac{\partial H}{\partial q_{i}} \frac{\partial\left(\vec{p}_{1}+\vec{p}_{2}\right)}{\partial p_{i}}=-\frac{\partial H}{\partial q_{i}} \frac{\partial(\vec{p})}{\partial p_{i}}=-\frac{\partial H}{\partial q_{i}} \hat{e}_{i},
$$

in which $\hat{e}_{i}$ is a unit vector along $\vec{p}_{i}$ and the index $i$ indicates coordinates. Therefore one can omit the six independent components of $\vec{p}_{1}$ and $\vec{p}_{2}$; $p_{1 x}, p_{1 y}, p_{1 z}, p_{2 x}, p_{2 y}$ and $p_{2 z}$.) terms $\frac{p_{1}^{2}}{2 m_{1}}$ and $\frac{p_{2}^{2}}{2 m_{2}}$ in (11), i.e.

As it is seen, the Poisson's bracket (12) contains only the derivatives of Hamiltonian with respect to the generalized

$$
H=\frac{q_{1} q_{2}}{4 \pi \varepsilon_{0} c^{3}} \frac{\vec{p}_{1}}{2 m_{1}} \cdot \frac{\vec{v}_{2}}{\left|\vec{r}_{2}-\vec{r}_{1}\right|}+\frac{1}{2 m_{1}}\left(\frac{q_{1} q_{2}}{4 \pi \varepsilon_{0} c^{3}}\right)^{2} \frac{\vec{v}_{2}^{2}}{\left|\vec{r}_{2}-\vec{r}_{1}\right|}+\frac{q_{1} q_{2}}{4 \pi \varepsilon_{0} c^{3}} \frac{\vec{p}_{2}}{2 m_{2}} \cdot \frac{\vec{v}_{1}}{\left|\vec{r}_{2}-\vec{r}_{1}\right|}+\frac{1}{2 m_{2}}\left(\frac{q_{1} q_{2}}{4 \pi \varepsilon_{0} c^{3}}\right)^{2} \frac{\vec{v}_{1}^{2}}{\left|\vec{r}_{2}-\vec{r}_{1}\right|}
$$

Substituting $\vec{p}_{1}=m_{1} \vec{v}_{1}$ and $\vec{p}_{2}=m_{2} \vec{v}_{2}$ in above expression we get:

$$
H=\frac{2 q_{1} q_{2}}{4 \pi \varepsilon_{0} c^{3}} \frac{\vec{v}_{1} \cdot \vec{v}_{2}}{\left|\vec{r}_{2}-\vec{r}_{1}\right|}+\frac{1}{2 m_{1}}\left(\frac{q_{1} q_{2}}{4 \pi \varepsilon_{0} c^{3}}\right)^{2} \frac{\vec{v}_{2}^{2}}{\left|\vec{r}_{2}-\vec{r}_{1}\right|}+\frac{1}{2 m_{2}}\left(\frac{q_{1} q_{2}}{4 \pi \varepsilon_{0} c^{3}}\right)^{2} \frac{\vec{v}_{1}^{2}}{\left|\vec{r}_{2}-\vec{r}_{1}\right|}
$$

Now in order to simplify our results, we notate 


$$
\begin{aligned}
& \frac{2 q_{1} q_{2}}{4 \pi \varepsilon_{0} c^{3}} \vec{v}_{1} \cdot \vec{v}_{2} \equiv k_{1}, \\
& \frac{1}{2 m_{1}}\left(\frac{q_{1} q_{2}}{4 \pi \varepsilon_{0} c^{3}}\right)^{2} \vec{v}_{2}^{2} \equiv k_{2}, \\
& \frac{1}{2 m_{2}}\left(\frac{q_{1} q_{2}}{4 \pi \varepsilon_{0} c^{3}}\right)^{2} \vec{v}_{1}^{2} \equiv k_{2}^{\prime} .
\end{aligned}
$$

Consequently (14) becomes

$$
H=\frac{k_{1}}{\left|\vec{r}_{2}-\vec{r}_{1}\right|}+\frac{k_{2}}{\left|\vec{r}_{2}-\vec{r}_{1}\right|^{2}}+\frac{k_{2}^{\prime}}{\left|\vec{r}_{2}-\vec{r}_{1}\right|^{2}} .
$$

\section{Discussion and Conclusion}

An interesting point in the above Hamiltonian is its symmetry with respect to exchanges between $\vec{r}_{1}$ and $\vec{r}_{2}$. Now expanding the Poisson's bracket (12) we get

$$
\begin{array}{r}
\left\{\vec{p}_{1}+\vec{p}_{2}, H\right\}=-\frac{\partial H}{\partial q_{i}} \hat{e}_{i}=-\frac{\partial H}{\partial x_{1}} \hat{e}_{1}-\frac{\partial H}{\partial y_{1}} \hat{e}_{2}-\frac{\partial H}{\partial z_{1}} \hat{e}_{3} \\
\frac{\partial H}{\partial x_{2}} \hat{e}_{1}-\frac{\partial H}{\partial y_{2}} \hat{e}_{2}-\frac{\partial H}{\partial z_{2}} \hat{e}_{3} .
\end{array}
$$

If the above differentiations are done with respect to the indexes 1 and 2 , then one observes that $\frac{\partial H}{\partial x_{1}}=-\frac{\partial H}{\partial x_{2}}, \ldots$. This is of course observable from the equation of symmetry. Hence we have

$$
\left\{\vec{p}_{1}+\vec{p}_{2}, H\right\}=0,
$$

or

$$
\frac{\mathrm{d}}{\mathrm{d} t}\left(\vec{p}_{1}+\vec{p}_{2}\right)=0 \quad \Rightarrow \quad \vec{p}_{1}+\vec{p}_{2}=\text { const. }
$$

which means that for a two-electron system (or for two charges $q_{1}$ and $q_{2}$ in general), the conservation of linear momentum is retained, despite the fact that they are not subjected to centripetal forces. Therefore once can observe that, when no central force is applied on charges, the linear momentum is still conserved. This interesting conclusion extends the usual domain of linear momentum conservation and this is what we were looking into in this paper.

\section{References}

[1] Herbert Goldstein: Classical mechanics (2nd ed.), Reading, Mass.: Addison-Wesley Pub. Co., ISBN 0201029189 (1980)

[2] John David Jackson: Classical electrodynamics (2nd ed.), New York: Wiley, ISBN 047143132X (1975)

[3] D. Halliday, R. Resnick, J. Walker: Fundamentals of Physics, John Wiley and Sons; 7th edition (June 14, 2004), ISBN-13: 978-0471216438

[4] A. Eden: The search for Christian Doppler, Springer-Verlag, Wien (1992)

[5] E. Adrian: Doppler Effect, NCSA (1995)

[6] B. Ballot: Annalen der Physik und Chemie 11: 321-351 (1845)

[7] Fizeau: Acoustique et optique, Lecture, Société Philomathique de Paris, 29 December , (1848)

[8] J. S. Russell: Report of the Eighteenth Meeting of the British Association for the Advancement of Science, London, 18, 7, 37-38 (1849)

[9] J. Rosen, L.Q. Gothard: Encyclopedia of Physical Science. Infobase Publishing. p. 155 (2009)

[10] K.R. Symon: Mechanics ( $3^{\text {rd }}$ edition), Addison-Wesley Pub. Co., ISBN 978-0201073928 (1971)

[11] S.T. Thomton: Classical Mechanics of Particles and Systems ( $5^{\text {th }}$ edition), Cengage Learning, ISBN 978-0534408961 (2003) 